\title{
Asymptotic Behavior of Solutions to Fast Diffusive Non-Newtonian Filtration Equations Coupled by Nonlinear Boundary Sources
}

\author{
Wang Zejia, ${ }^{1}$ Wang Shunti, ${ }^{2}$ and Zhang Chengbin ${ }^{2}$ \\ ${ }^{1}$ College of Mathematics and Information Science, Jiangxi Normal University, Nanchang 330022, China \\ ${ }^{2}$ Institute of Mathematics, Jilin University, Changchun 130012, China \\ Correspondence should be addressed to Wang Zejia; wangzj1979@gmail.com
}

Received 6 February 2013; Accepted 27 March 2013

Academic Editor: Sining Zheng

Copyright (C) 2013 Wang Zejia et al. This is an open access article distributed under the Creative Commons Attribution License, which permits unrestricted use, distribution, and reproduction in any medium, provided the original work is properly cited.

This paper is concerning the asymptotic behavior of solutions to the fast diffusive non-Newtonian filtration equations coupled by the nonlinear boundary sources. We are interested in the critical global existence curve and the critical Fujita curve, which are used to describe the large-time behavior of solutions. It is shown that the above two critical curves are both the same for the multidimensional problem we considered.

\section{Introduction}

In this paper, we study the non-Newtonian filtration equations coupled by the nonlinear boundary sources

$$
\begin{aligned}
& \frac{\partial u}{\partial t}=\operatorname{div}\left(|\nabla u|^{p-2} \nabla u\right), \frac{\partial v}{\partial t}=\operatorname{div}\left(|\nabla v|^{q-2} \nabla v\right), \\
&(x, t) \in\left(\mathbb{R}^{N} \backslash B_{1}(0)\right) \times(0, T), \\
&|\nabla u|^{p-2} \nabla u \cdot \vec{v}=v^{\alpha}(x, t),|\nabla v|^{q-2} \nabla v \cdot \vec{v}=u^{\beta}(x, t), \\
&(x, t) \in \partial B_{1}(0) \times(0, T), \\
& u(x, 0)=u_{0}(x), \quad v(x, 0)=v_{0}(x), \quad x \in \mathbb{R}^{N} \backslash B_{1}(0),
\end{aligned}
$$

where $1<p, q<2, \alpha, \beta \geq 0, N \geq 2, B_{1}(0)$ is the unit ball in $\mathbb{R}^{N}$ with boundary $\partial B_{1}(0), \vec{v}$ is the inward normal vector on $\partial B_{1}(0)$, and $u_{0}(x)$ and $v_{0}(x)$ are nonnegative, suitably smooth, and bounded functions satisfying the appropriate compatibility conditions.

The system (1)-(3) can be used to describe the models in population dynamics, chemical reactions, heat propagation, and so on. It is well known that the classical solutions do not exist because the equations in (1) are degenerate in $\{(x, t) ; \nabla u(x, t)=0\}$, while the local existence and the comparison principle of the weak solutions can be obtained; see $[1,2]$. In this paper, we investigate the asymptotic behavior of solutions to the system (1)-(3), including blowup in a finite time and global existence in time.

Since the beginning work on critical exponent done by Fujita in [3], there are a lot of Fujita type results established for various equations; see the survey papers $[4,5]$ and the references therein and also the papers [6-9]. We recall some results on the fast diffusion case. In [10], the authors obtained the critical exponents for the single one-dimensional fast diffusive equation on $(0,+\infty) \times(0,+\infty)$, that is,

$$
\begin{gathered}
\frac{\partial u}{\partial t}=\frac{\partial}{\partial x}\left(\left|\frac{\partial u}{\partial x}\right|^{p-2} \frac{\partial u}{\partial x}\right), \quad x>0, t>0, \\
\left|\frac{\partial u}{\partial x}\right|^{p-2} \frac{\partial u}{\partial x}(0, t)=u^{\alpha}(0, t), \quad t>0 \\
u(x, 0)=u_{0}(x), \quad x>0 .
\end{gathered}
$$

They showed that the global critical exponent is $\alpha_{0}=2(p-$ 1) $/ p$ and the critical Fujita exponent is $\alpha_{c}=2(p-1)$. 
After that, the corresponding results on the coupled $p$-laplace equations can be found in the paper [11], in which the authors consider multiple equations coupled by boundary sources. For the system,

$$
\begin{gathered}
\frac{\partial u}{\partial t}=\frac{\partial}{\partial x}\left(\left|\frac{\partial u}{\partial x}\right|^{p-2} \frac{\partial u}{\partial x}\right), \\
\frac{\partial v}{\partial t}=\frac{\partial}{\partial x}\left(\left|\frac{\partial v}{\partial x}\right|^{q-2} \frac{\partial v}{\partial x}\right), \quad x>0, t>0, \\
\left|\frac{\partial u}{\partial x}\right|^{p-2} \frac{\partial u}{\partial x}(0, t)=v^{\alpha}(0, t), \\
\left|\frac{\partial v}{\partial x}\right|^{q-2} \frac{\partial u}{\partial x}(0, t)=u^{\beta}(0, t), \quad t>0, \\
u(x, 0)=u_{0}(x), \quad v(x, 0)=v_{0}(x), \quad x>0,
\end{gathered}
$$

the results in [11] are that the critical global existence curve is $\alpha \beta=4(p-1)(q-1) /(p q)$, and when $\alpha \beta>4(p-1)(q-1) /(p q)$, the critical Fujita curve is $\min \left\{l_{1}-k_{1}, l_{2}-k_{2}\right\}=0$, where $k_{1}, k_{2}, l_{1}$, and $l_{2}$ are constants depending on $p, q, \alpha$, and $\beta$. It can be seen by some computations that the critical Fujita curve is on the strictly right of the critical global existence curve.

As for the multidimensional problem, the single equation case of (1)-(3) was discussed in [12]; that is,

$$
\begin{gathered}
\frac{\partial u}{\partial t}=\operatorname{div}\left(|\nabla u|^{p-2} \nabla u\right), \quad(x, t) \in\left(\mathbb{R}^{N} \backslash B_{1}(0)\right) \times(0, T) \\
|\nabla u|^{p-2} \nabla u \cdot \vec{v}=u^{\alpha}(x, t), \quad(x, t) \in \partial B_{1}(0) \times(0, T) \\
u(x, 0)=u_{0}(x), \quad x \in \mathbb{R}^{N} \backslash B_{1}(0)
\end{gathered}
$$

with $1<p<2, \alpha \geq 0, N \geq 2$. It was shown that the critical global exponent and the critical Fujita exponent both are $\alpha=$ $p-1$.

Motivated by the papers mentioned above, the aim of this paper is to study the asymptotic behavior of solutions to the system (1)-(3). We show that the phenomenon that the two critical exponents for multi-dimensional equation coincide also occurs in the coupled equations.

Furthermore, by virtue of the radial symmetry of the exterior domain of the unit ball, we note that the above result can be extended to the following more general problems:

$$
\begin{array}{r}
|\nabla u|^{p-2} \nabla u \cdot \vec{v}=v^{\alpha}(x, t), \quad|\nabla v|^{q-2} \nabla v \cdot \vec{v}=u^{\beta}(x, t), \\
(x, t) \in \partial B_{1}(0) \times(0, T), \\
u(x, 0)=u_{0}(x), \quad v(x, 0)=v_{0}(x), \quad x \in \mathbb{R}^{N} \backslash B_{1}(0),
\end{array}
$$

with $\lambda_{1}>p-N, \lambda_{2}>q-N, N \geq 1$.

We will state our main results and prove them in the next section.

\section{Main Results and Their Proofs}

In this section, we first state our main results and then prove them. Our main results are as follows.

Theorem 1. The critical global existence curve and the critical Fujita curve for the system (1)-(3) with $N \geq 2$ are the same one, that is, the curve given by

$$
\alpha \beta=\frac{4(p-1)(q-1)}{p q} .
$$

Namely, if $\alpha \beta \leq 4(p-1)(q-1) /(p q)$, then all nonnegative solutions of the system (1)-(3) exist globally in time, while if $\alpha \beta>4(p-1)(q-1) /(p q)$, then there exist both blow-up solutions for large initial data and global existent solution for small initial data.

Theorem 2. Assume that $\lambda_{1}>p-N, \lambda_{2}>q-N$, and $N \geq$ 1. Then both the critical global existence curve and the critical Fujita curve for the system (7)-(9) are the curve given by

$$
\alpha \beta=\frac{4(p-1)(q-1)}{p q} .
$$

Since the proofs of Theorems 1 and 2 are similar, we shall give the proof of Theorem 2 only. We note that the equations in (1) and (7) are degenerate at the points where $\nabla u(x, t)=0$, and classical solutions may not exist generally. It is therefore necessary to consider weak solutions in the distribution sense.

To prove Theorem 2, we first establish some preliminary results. Let $r=|x|$ and consider the problem:

$$
\begin{gathered}
\frac{\partial u}{\partial t}=\frac{\partial}{\partial r}\left(\left|\frac{\partial u}{\partial r}\right|^{p-2} \frac{\partial u}{\partial r}\right)+\frac{\tilde{\lambda}_{1}}{r}\left|\frac{\partial u}{\partial r}\right|^{p-2} \frac{\partial u}{\partial r}, \\
\frac{\partial v}{\partial t}=\frac{\partial}{\partial r}\left(\left|\frac{\partial v}{\partial r}\right|^{q-2} \frac{\partial v}{\partial r}\right)+\frac{\tilde{\lambda}_{2}}{r}\left|\frac{\partial v}{\partial r}\right|^{q-2} \frac{\partial v}{\partial r}, \quad r>1, \quad t>0
\end{gathered}
$$

$$
\begin{gathered}
\left|\frac{\partial u}{\partial r}\right|^{p-2} \frac{\partial u}{\partial r}(1, t)=v^{\alpha}(1, t), \\
\left|\frac{\partial v}{\partial r}\right|^{q-2} \frac{\partial v}{\partial r}(1, t)=u^{\beta}(1, t), \quad t>0, \\
u(r, 0)=u_{0}(r), \quad v(r, 0)=v_{0}(r), \quad r>1,
\end{gathered}
$$


where $1<p, q<2, \tilde{\lambda}_{1}=\lambda_{1}+N-1>p-1, \tilde{\lambda}_{2}>$ $q-1, \alpha, \beta \geq 0, u_{0}, v_{0}$ are nonnegative, suitably smooth, and bounded functions. It is not difficult to check that the solution $(u, v)(r, t)$ of the system (12)-(14) is also the solution of the system (7)-(9) if $u_{0}(x)$ and $v_{0}(x)$ are radially symmetrical. We now study the asymptotic behavior of solutions to the system (12)-(14).

Proposition 3. If $\alpha \beta \leq 4(p-1)(q-1) / p q$, then all nonnegative solutions of the system (12)-(14) exist globally in time.

Proof of Proposition 3. We prove this proposition by constructing a kind of global upper solutions. Let

$$
\begin{array}{lll}
\bar{u}(r, t)=\mathrm{e}^{L_{1} t}\left(K+\mathrm{e}^{-M_{1} \eta_{1}}\right), & \eta_{1}=(r-1) \mathrm{e}^{J_{1} t}, & r>1, t>0, \\
\bar{v}(r, t)=\mathrm{e}^{L_{2} t}\left(K+\mathrm{e}^{-M_{2} \eta_{2}}\right), & \eta_{2}=(r-1) \mathrm{e}^{J_{2} t}, & r>1, t>0,
\end{array}
$$

where $M_{1}=(K+1)^{\alpha /(p-1)}, M_{2}=(K+1)^{\beta /(q-1)}, J_{1}=L_{1}(2-$ $p) / p, J_{2}=L_{2}(2-q) / q, L_{2}=2(p-1) L_{1} /(p \alpha)$, and $K$ and $L_{1}$ are large constants satisfying that

$$
\begin{aligned}
& K>\max \left\{1,\left\|u_{0}\right\|_{\infty},\left\|v_{0}\right\|_{\infty}, \frac{2-p}{p \mathrm{e}}, \frac{2-q}{q \mathrm{e}}\right\}, \\
& L_{1}>\max \left\{\frac{p(p-1) e M_{1}^{p}}{p-2+p e K}, \frac{\alpha p q(q-1) e M_{2}^{q}}{2(p-1)(q-2+q e K)}\right\} .
\end{aligned}
$$

Obviously, we have $\bar{u}(r, 0) \geq u_{0}(r)$ and $\bar{v}(r, 0) \geq v_{0}(r)$ for $r>1$, and a direct computation yields that

$$
\begin{aligned}
\left|\frac{\partial \bar{u}}{\partial r}\right|^{p-2} \frac{\partial \bar{u}}{\partial r} & =-M_{1}^{p-1} \mathrm{e}^{(p-1)\left(L_{1}+J_{1}\right) t} \mathrm{e}^{-(p-1)(r-1) M_{1} \mathrm{e}^{J_{1} t}}<0, \\
\frac{\partial}{\partial r}\left(\left|\frac{\partial \bar{u}}{\partial r}\right|^{p-2} \frac{\partial \bar{u}}{\partial r}\right) & =(p-1) M_{1}^{p} \mathrm{e}^{\left((p-1) L_{1}+p J_{1}\right) t} \mathrm{e}^{-(p-1)(r-1) M_{1} \mathrm{e}^{J_{1}} t} \\
& \leq(p-1) M_{1}^{p} \mathrm{e}^{\left((p-1) L_{1}+p J_{1}\right) t} .
\end{aligned}
$$

Noticing that $-y \mathrm{e}^{-y} \geq-\mathrm{e}^{-1}$ for $y>0$, we have

$$
\begin{aligned}
\frac{\partial \bar{u}}{\partial t}= & L_{1} \mathrm{e}^{L_{1} t}\left(K+\mathrm{e}^{-M_{1}(r-1) \mathrm{e}^{J_{1} t}}\right) \\
& +J_{1} \mathrm{e}^{L_{1} t}\left(-M_{1}(r-1) \mathrm{e}^{J_{1} t} \mathrm{e}^{-M_{1}(r-1) \mathrm{e}^{J_{1} t}}\right) \\
\geq & L_{1} \mathrm{e}^{L_{1} t}\left(K+\mathrm{e}^{-M_{1}(r-1) \mathrm{e}^{J_{1} t}}\right)-J_{1} \mathrm{e}^{L_{1} t} \mathrm{e}^{-1} \\
\geq & \left(L_{1}-\frac{J_{1}}{K \mathrm{e}}\right) K \mathrm{e}^{L_{1} t} .
\end{aligned}
$$

Due to the choice of $L_{1}$ and $(p-1) L_{1}+p J_{1}=L_{1}, \widetilde{\lambda}_{1}>0$, then we get

$$
\begin{aligned}
\frac{\partial \bar{u}}{\partial t} & \geq \frac{\partial}{\partial r}\left(\left|\frac{\partial \bar{u}}{\partial r}\right|^{p-2} \frac{\partial \bar{u}}{\partial r}\right) \\
& \geq \frac{\partial}{\partial r}\left(\left|\frac{\partial \bar{u}}{\partial r}\right|^{p-2} \frac{\partial \bar{u}}{\partial r}\right)+\frac{\tilde{\lambda}_{1}}{r}\left|\frac{\partial \bar{u}}{\partial r}\right|^{p-2} \frac{\partial \bar{u}}{\partial r} .
\end{aligned}
$$

On the other hand,

$$
\begin{aligned}
-\left|\frac{\partial \bar{u}}{\partial r}\right|^{p-2} \frac{\partial \bar{u}}{\partial r}(1, t) & =M_{1}^{p-1} \mathrm{e}^{(p-1)\left(L_{1}+J_{1}\right) t} \\
& =(K+1)^{\alpha} \mathrm{e}^{2(p-1) L_{1} t / p}, \\
\bar{v}^{\alpha}(1, t) & =\mathrm{e}^{\alpha L_{2} t}(K+1)^{\alpha} \\
& =(K+1)^{\alpha} \mathrm{e}^{2(p-1) L_{1} t / p} \\
& =-\left|\frac{\partial \bar{u}}{\partial x}\right|^{p-2} \frac{\partial \bar{u}}{\partial x}(1, t) .
\end{aligned}
$$

Similarly, we have

$$
\begin{aligned}
& \frac{\partial \bar{v}}{\partial t} \geq \frac{\partial}{\partial r}\left(\left|\frac{\partial \bar{v}}{\partial r}\right|^{q-2} \frac{\partial \bar{v}}{\partial r}\right)+\frac{\tilde{\lambda}_{2}}{r}\left|\frac{\partial \bar{v}}{\partial r}\right|^{q-2} \frac{\partial \bar{v}}{\partial r} \\
&-\left|\frac{\partial \bar{v}}{\partial r}\right|^{q-2} \frac{\partial \bar{v}}{\partial r}(1, t)=M_{2}^{q-1} \mathrm{e}^{(q-1)\left(L_{2}+J_{2}\right) t} \\
&=(K+1)^{\beta} \mathrm{e}^{4(p-1)(q-1) L_{1} t /(p q \alpha)} .
\end{aligned}
$$

Since that $\alpha \beta \leq 4(p-1)(q-1) /(p q)$, then

$$
\bar{u}^{\beta}(1, t)=\mathrm{e}^{\beta L_{1} t}(K+1)^{\beta} \leq(K+1)^{\beta} \mathrm{e}^{4(p-1)(q-1) L_{1} t /(p q \alpha)} .
$$

This indicates that

$$
-\left|\frac{\partial \bar{v}}{\partial r}\right|^{q-2} \frac{\partial \bar{v}}{\partial r}(1, t) \geq \bar{u}^{\beta}(1, t) .
$$

Noticing the global existence in time of $(\bar{u}, \bar{v})$, we get that the solution of the problem (12)-(14) exists globally by the comparison principle. The proof is complete.

Proposition 4. If $\alpha \beta>4(p-1)(q-1) /(p q)$, then the nonnegative nontrivial solutions of the system (12)-(14) blow up in finite time for large initial data.

Proof of Proposition 4. The proposition is proved by constructing a kind of lower blow-up solutions. Set

$$
\begin{gathered}
\underline{u}(r, t)=(T-t)^{-k_{1}} f_{1}(\xi), \\
\xi=(r-1)(T-t)^{-l_{1}}, \quad r>1, t>0, \\
\underline{v}(r, t)=(T-t)^{-k_{2}} f_{2}(\eta), \\
\eta=(r-1)(T-t)^{-l_{2}}, \quad r>1, t>0,
\end{gathered}
$$

where $T>0$, and

$$
\begin{gathered}
k_{1}=\frac{(q-1)(2(p-1)+\alpha p)}{\alpha \beta p q-4(p-1)(q-1)}, \quad l_{1}=\frac{1+(2-p) k_{1}}{p}, \\
k_{2}=\frac{(p-1)(2(q-1)+\beta q)}{\alpha \beta p q-4(p-1)(q-1)}, \quad l_{2}=\frac{1+(2-q) k_{2}}{q} .
\end{gathered}
$$


Due to $1<p, q<2, \alpha \beta>4(p-1)(q-1) /(p q)$, we see that $k_{1}, l_{1}, k_{2}, l_{2}>0$ and

$$
\begin{array}{ll}
k_{1}+1=(p-1) k_{1}+p l_{1}, & (p-1)\left(k_{1}+l_{1}\right)=k_{2} \alpha, \\
k_{2}+1=(q-1) k_{2}+q l_{2}, & (q-1)\left(k_{2}+l_{2}\right)=k_{1} \beta .
\end{array}
$$

We claim that $(\underline{u}, \underline{v})$ is a lower solution to the problem (12)-(14) with $u_{0}(r) \geq \underline{u}(r, 0), v_{0}(r) \geq \underline{v}(r, 0)$ if the following inequalities hold:

$$
\begin{gathered}
\left(\left|f_{1}^{\prime}(\xi)\right|^{p-2} f_{1}^{\prime}(\xi)\right)^{\prime}+\frac{\tilde{\lambda}_{1}(T-t)^{l_{1}}}{r}\left|f_{1}^{\prime}(\xi)\right|^{p-2} f_{1}^{\prime}(\xi) \\
-l_{1} \xi f_{1}^{\prime}(\xi)-k_{1} f_{1}(\xi) \geq 0, \\
-\left|f_{1}^{\prime}(0)\right|^{p-2} f_{1}^{\prime}(0) \leq f_{2}^{\alpha}(0), \\
\left(\left|f_{2}^{\prime}(\eta)\right|^{q-2} f_{2}^{\prime}(\eta)\right)^{\prime}+\frac{\tilde{\lambda}_{2}(T-t)^{l_{2}}}{r}\left|f_{2}^{\prime}(\eta)\right|^{q-2} f_{2}^{\prime}(\eta) \\
-l_{2} \eta f_{2}^{\prime}(\eta)-k_{2} f_{2}(\eta) \geq 0, \\
-\left|f_{2}^{\prime}(0)\right|^{q-2} f_{2}^{\prime}(0) \leq f_{1}^{\beta}(0) .
\end{gathered}
$$

Note that

$$
\begin{aligned}
& \frac{\tilde{\lambda}_{1}(T-t)^{l_{1}}}{r} \leq \tilde{\lambda}_{1}(T-t)^{l_{1}} \leq \tilde{\lambda}_{1} T^{l_{1}}, \\
& \frac{\tilde{\lambda}_{2}(T-t)^{l_{1}}}{r} \leq \tilde{\lambda}_{2} T^{l_{2}}, \quad \text { for } r>1 .
\end{aligned}
$$

So if we assume that $f_{1}, f_{2}$ satisfy

$$
\begin{gathered}
f_{1}(\xi), f_{2}(\eta) \geq 0, \quad f_{1}(\xi)^{\prime}, f_{2}(\eta)^{\prime} \leq 0, \\
f_{1}(\xi)^{\prime \prime}, f_{2}(\eta)^{\prime \prime} \geq 0, \quad \xi, \eta \geq 0,
\end{gathered}
$$

then (27)- (30) hold provided that

$$
\begin{gathered}
\left(\left|f_{1}^{\prime}(\xi)\right|^{p-2} f_{1}^{\prime}(\xi)\right)^{\prime}+\tilde{\lambda}_{1} T l_{1}\left|f_{1}^{\prime}(\xi)\right|^{p-2} f_{1}^{\prime}(\xi)-k_{1} f_{1}(\xi) \geq 0 \\
-\left|f_{1}^{\prime}(0)\right|^{p-2} f_{1}^{\prime}(0) \leq f_{2}^{\alpha}(0) \\
\left(\left|f_{2}^{\prime}(\eta)\right|^{q-2} f_{2}^{\prime}(\eta)\right)^{\prime} \\
+\tilde{\lambda}_{2} T^{l_{2}}\left|f_{2}^{\prime}(\eta)\right|^{q-2} f_{2}^{\prime}(\eta)-k_{2} f_{2}(\eta) \geq 0 \\
-\left|f_{2}^{\prime}(0)\right|^{q-2} f_{2}^{\prime}(0) \leq f_{1}^{\beta}(0)
\end{gathered}
$$

Take

$$
\begin{aligned}
& f_{1}(\xi)=\left(\left(A_{1}+B_{1} \xi\right)^{p /(p-2)}-\left(2 A_{1}\right)^{p /(p-2)}\right)_{+}, \quad \xi \geq 0, \\
& f_{2}(\eta)=\left(\left(A_{2}+B_{2} \eta\right)^{q /(q-2)}-\left(2 A_{2}\right)^{q /(q-2)}\right)_{+}, \quad \eta \geq 0,
\end{aligned}
$$

where $0<A_{1}, A_{2}<1, B_{1}, B_{2}>0$ are the constants to be determined. It is clear that the above $f_{1}, f_{2}$ satisfy (32). Now, we verify that $f_{1}, f_{2}$ satisfy (33)-(36) in the distribution sense. We claim that (33) is valid for $0<\xi<A_{1} / B_{1}$. In fact, we only need to verify that

$$
\begin{aligned}
& \frac{1}{2}\left(\left|f_{1}^{\prime}(\xi)\right|^{p-2} f_{1}^{\prime}(\xi)\right)^{\prime} \geq-\widetilde{\lambda}_{1} T^{l_{1}}\left|f_{1}^{\prime}(\xi)\right|^{p-2} f_{1}^{\prime}(\xi), \\
& \frac{1}{2}\left(\left|f_{1}^{\prime}(\xi)\right|^{p-2} f_{1}^{\prime}(\xi)\right)^{\prime} \geq k_{1} f_{1}(\xi),
\end{aligned}
$$

that is,

$$
\begin{aligned}
\frac{p-1}{2} f_{1}^{\prime \prime}(\xi) & \geq-\tilde{\lambda}_{1} T^{l_{1}} f_{1}^{\prime}(\xi), \\
\frac{p-1}{2}\left|f_{1}^{\prime}(\xi)\right|^{p-2} f_{1}^{\prime \prime}(\xi) & \geq k_{1} f_{1}(\xi) .
\end{aligned}
$$

In the first place, we compute each term in (40) as follows:

$$
\begin{gathered}
\frac{p-1}{2} f_{1}^{\prime \prime}(\xi)=\frac{p-1}{2} \frac{2 p B_{1}^{2}}{(p-2)^{2}}\left(A_{1}+B_{1} \xi\right)^{(4-p) /(p-2)}, \\
-\widetilde{\lambda}_{1} T^{l_{1}} f_{1}^{\prime}(\xi)=-\widetilde{\lambda}_{1} T^{l_{1}} \frac{p B_{1}}{p-2}\left(A_{1}+B_{1} \xi\right)^{2 /(p-2)}, \\
\frac{p-1}{2}\left|f_{1}^{\prime}(\xi)\right|^{p-2} f_{1}^{\prime \prime}(\xi)=\frac{(p-1) p^{p-1} B_{1}^{p}}{(2-p)^{p}}\left(A_{1}+B_{1} \xi\right)^{p /(p-2)}, \\
k_{1} f_{1}(\xi)=k_{1}\left(\left(A_{1}+B_{1} \xi\right)^{p /(p-2)}-\left(2 A_{1}\right)^{p /(p-2)}\right) .
\end{gathered}
$$

Then, the inequalities in (40) are valid provided that, for $0<$ $\xi<A_{1} / B_{1}$,

$$
\begin{gathered}
\frac{(p-1) B_{1}}{2-p} \geq 2 \widetilde{\lambda}_{1} A_{1} T^{l_{1}} \geq \widetilde{\lambda}_{1} T^{l_{1}}\left(A_{1}+B_{1} \xi\right), \\
\frac{(p-1) p^{p-1} B_{1}^{p}}{(2-p)^{p}} \geq k_{1} .
\end{gathered}
$$

So, we choose $B_{1}=k_{1}^{1 / p}(2-p) /\left[(p-1) p^{p-1}\right]^{1 / p}$, and $A_{1}$ is small enough that

$$
A_{1} \leq \frac{B_{1}(p-1)}{\tilde{\lambda}_{1} T^{l_{1}}(2-p)} .
$$

Similarly, choose $B_{2}, A_{2}$, satisfying that

$$
B_{2}=\left(k_{2} \frac{(2-q)^{q}}{(q-1) q^{q-1}}\right)^{1 / q}, \quad A_{2} \leq \frac{B_{2}(q-1)}{\tilde{\lambda}_{2} T^{l_{2}}(2-q)}
$$

to get the validity of (35). 
Next, we verify that $f_{1}$ and $f_{2}$ given by (37) and (38) also satisfy the boundary conditions (34) and (36):

$$
\begin{aligned}
-\left|f_{1}^{\prime}(0)\right|^{p-2} f_{1}^{\prime}(0) & =\left(\frac{p B_{1}}{2-p} A_{1}^{2 /(p-2)}\right)^{p-1} \\
& =\left(\frac{p B_{1}}{2-p}\right)^{p-1} A_{1}^{2(p-1) /(p-2)}, \\
f_{2}^{\alpha}(0) & =\left(A_{2}^{q / q-2}-\left(2 A_{2}\right)^{q / q-2}\right)^{\alpha} \\
& =\left(1-2^{q / q-2}\right)^{\alpha} A_{2}^{q \alpha /(q-2)} .
\end{aligned}
$$

From (34), we need

$$
\left(\frac{p B_{1}}{2-p}\right)^{p-1} A_{1}^{2(p-1) /(p-2)} \leq\left(1-2^{q /(q-2)}\right)^{\alpha} A_{2}^{q \alpha /(q-2)} .
$$

Set $A_{2}=A_{1}^{\sigma}$, where $\sigma$ is to be determined. Then rewriting the last inequality, we get

$$
A_{1}^{2(p-1) /(p-2)-q \alpha \sigma /(q-2)} \leq\left(1-2^{q /(q-2)}\right)^{\alpha}\left(\frac{2-p}{p B_{1}}\right)^{p-1}
$$

which can be obtained by choosing $A_{1}>0$ small enough, if

$$
\frac{2(p-1)}{p-2}-\frac{q \alpha \sigma}{q-2}>0
$$

In a similar discussion on the boundary condition (36), for enough small $A_{1}$, the following inequality is needed:

$$
\frac{2 \sigma(q-1)}{q-2}-\frac{p \beta}{p-2}>0 .
$$

Note that $1<p$ and $q<2$; therefore, (48)-(49) are equal to

$$
\frac{2(p-1)}{q \alpha}<\sigma \frac{p-2}{q-2}<\frac{p \beta}{2(q-1)} .
$$

Recall the assumption that $\alpha \beta>4(p-1)(q-1) / p q$, so there exists a constant $\sigma>0$, such that (50) holds. Furthermore, we can choose $A_{1}$ as small as needed.

Therefore, the solution $(u, v)$ of the problem (12)-(14) blows up in a finite time if $\left(u_{0}(r), v_{0}(r)\right)$ is large enough such that

$$
u_{0}(r) \geq \underline{u}(r, 0), \quad v_{0}(r) \geq \underline{v}(r, 0), \quad r>1 .
$$

The proof is completed.

Proposition 5. If $\alpha \beta \neq(p-1)(q-1)$, then every nonnegative nontrivial solution of the system (12)-(14) with small initial data exists globally.

Proof of Proposition 5. We seek the steady-state solution of the system (12)-(14):

$$
u(r, t)=\widetilde{u}(r), \quad v(r, t)=\widetilde{v}(r), \quad r>1, t>0 .
$$

A direct calculation shows that $\widetilde{u}(r), \widetilde{v}(r)$ should satisfy

$$
\begin{gathered}
\left(r^{\tilde{\lambda}_{1}}\left|\tilde{u}^{\prime}\right|^{p-2} \widetilde{u}^{\prime}\right)^{\prime}=0, \quad\left(r^{\tilde{\lambda}_{2}}\left|\widetilde{v}^{\prime}\right|^{q-2} \widetilde{v}^{\prime}\right)^{\prime}=0, \quad r>1, \\
-\left|\tilde{u}^{\prime}(1)\right|^{p-2} \tilde{u}^{\prime}(1)=\widetilde{v}^{\alpha}(1), \quad-\left|\widetilde{v}^{\prime}(1)\right|^{p-2} \widetilde{v}^{\prime}(1)=\tilde{u}^{\beta}(1),
\end{gathered}
$$

which implies that

$$
\tilde{u}^{\prime}=-A_{2}^{\alpha /(p-1)} r^{-\tilde{\lambda}_{1} /(p-1)}, \quad \tilde{v}^{\prime}=-A_{1}^{\beta /(q-1)} r^{-\tilde{\lambda}_{2} /(q-1)}, \quad r>1
$$

with $A_{1}=\widetilde{u}(1), A_{2}=\widetilde{v}(1)$. Integrating the above equalities yields

$$
\begin{aligned}
\widetilde{u}(r)= & \left(A_{1}-\frac{A_{2}^{\alpha /(p-1)}}{\widetilde{\lambda}_{1} /(p-1)-1}\right) \\
& +\frac{A_{2}^{\alpha /(p-1)}}{\widetilde{\lambda}_{1} /(p-1)-1} r^{1-\widetilde{\lambda}_{1} /(p-1)}, \\
\widetilde{v}(r)= & \left(A_{2}-\frac{A_{1}^{\beta /(q-1)}}{\widetilde{\lambda}_{2} /(q-1)-1}\right) \\
& +\frac{A_{1}^{\beta /(q-1)}}{\widetilde{\lambda}_{2} /(q-1)-1} r^{1-\lambda 2 /(q-1)} .
\end{aligned}
$$

In particular, we let

$$
A_{1}-\frac{A_{2}^{\alpha /(p-1)}}{\tilde{\lambda}_{1} /(p-1)-1}=0, \quad A_{2}-\frac{A_{1}^{\beta /(q-1)}}{\tilde{\lambda}_{2} /(q-1)-1}=0 .
$$

Define $C_{1}=\tilde{\lambda}_{1} /(p-1)-1, C_{2}=\tilde{\lambda}_{2} /(q-1)-1$, and then $C_{1}>0, C_{2}>0$. Noticing that $\alpha \beta \neq(p-1)(q-1)$, we get

$$
\begin{aligned}
& A_{2}=\left(C_{1}^{\beta /(q-1)} C_{2}\right)^{(p-1)(q-1) /((p-1)(q-1)-\alpha \beta)}, \\
& A_{1}=C_{1} A_{2}^{\alpha /(p-1)} .
\end{aligned}
$$

Therefore, the bounded positive functions:

$$
\tilde{u}(r)=\frac{A_{2}^{\alpha /(p-1)}}{C_{1}} r^{-C_{1}}, \quad \tilde{v}(r)=\frac{A_{1}^{\beta /(q-1)}}{C_{2}} r^{-C_{2}}
$$

are just a couple of steady-state solutions of the problem (12)-(14) with the initial data $\widetilde{u}(r), \widetilde{v}(r)$. By the comparison principle, for any initial data $u_{0}(r), v_{0}(r)$ which is small enough to satisfy

$$
u_{0}(r) \leq \tilde{u}(r), \quad v_{0}(r) \leq \widetilde{v}(r), \quad r>1,
$$

the solutions of the problem (12)-(14) exist globally in time. 
Remark 6. Due to the fact that $(p-1)(q-1)<4(p-1)(q-$ $1) /(p q)$ for $1<p, q<2$, it is seen from Propositions 3 and 5 that all nonnegative solutions to the system (12)-(14) with enough small initial data exist globally in time.

Now, we prove the main result for the system (7)-(9), that is, Theorem 2.

Proof of Theorem 2. Noticing that the functions $u_{0}(x), v_{0}(x)$ are bounded, we can choose two bounded, radially symmetrical functions denoted by $u_{1}(x), v_{1}(x)$ satisfying that $u_{1}(x)=$ $u_{1}(|x|) \geq u_{0}(x), v_{1}(x)=v_{1}(|x|) \geq v_{0}(x)$, respectively. By using Proposition 3 and the comparison principle, we can obtain the global existence of solutions to the system (7)-(9).

For the initial data $\left(u_{0}, v_{0}\right)$ is large enough such that $u_{0}(x) \geq \underline{u}(|x|, 0), v_{0}(x) \geq \underline{v}(|x|, 0)$, here $\underline{u}(|x|, 0), \underline{v}(|x|, 0)$ are defined in the proof of Proposition 4 if $\alpha \beta>4(p-1)(q-$ $1) /(p q)$, then the solutions of the system (7)-(9) with such $\left(u_{0}, v_{0}\right)$ blow up in a finite time by the comparison principle and Proposition 4.

On the other hand, using the comparison principle again and combining with Proposition 5, we see that the solution $(u, v)$ of $(7)-(9)$ exists globally if

$$
\begin{aligned}
& u_{0}(x) \leq \frac{(p-1) A_{2}^{\alpha /(p-1)}}{\lambda_{1}+N-p}|x|^{-\left(\lambda_{1}+N-p\right) /(p-1)}, \\
& v_{0}(x) \leq \frac{(q-1) A_{1}^{\beta /(q-1)}}{\lambda_{2}+N-q}|x|^{-\left(\lambda_{2}+N-q\right) /(q-1)},
\end{aligned}
$$

where

$$
\begin{aligned}
A_{2}= & \left(\left(\frac{\lambda_{1}+N-p}{p-1}\right)^{\beta /(q-1)}\right. \\
& \left.\times\left(\frac{\lambda_{2}+N-q}{q-1}\right)\right)^{(p-1)(q-1) /[(p-1)(q-1)-\alpha \beta]}, \\
A_{1}= & \frac{\lambda_{1}+N-p}{p-1} A_{2}^{\alpha /(p-1)} .
\end{aligned}
$$

The proof is complete.

\section{Acknowledgments}

The authors would like to express their many thanks to the Editor and Reviewers for their constructive suggestions to improve the previous version of this paper. This work was supported by the NNSF.

\section{References}

[1] A. S. Kalashnikov, "Some problems of the qualitative theory of nonlinear degenerate second-order parabolic equations," Akademiya Nauk SSSR i Moskovskoe Matematicheskoe Obshchestvo, vol. 42, no. 2, pp. 169-222, 1987.

[2] Z. Wu, J. Zhao, J. Yin, and H. Li, Nonlinear Diffusion Equations, World Scientific Publishing, River Edge, NJ, USA, 2001.
[3] H. Fujita, "On the blowing up of solutions of the Cauchy problem for $u_{t}=\Delta u+u^{1+\alpha}$," Journal of the Faculty of Science. University of Tokyo. Section IA. Mathematics, vol. 13, pp. 109-124, 1966.

[4] K. Deng and H. A. Levine, "The role of critical exponents in blow-up theorems: the sequel," Journal of Mathematical Analysis and Applications, vol. 243, no. 1, pp. 85-126, 2000.

[5] V. A. Galaktionov and H. A. Levine, "A general approach to critical Fujita exponents in nonlinear parabolic problems," Nonlinear Analysis, vol. 34, no. 7, pp. 1005-1027, 1998.

[6] R. Ferreira, A. de Pablo, F. Quirós, and J. D. Rossi, “The blow-up profile for a fast diffusion equation with a nonlinear boundary condition," The Rocky Mountain Journal of Mathematics, vol. 33, no. 1, pp. 123-146, 2003.

[7] F. Quirós and J. D. Rossi, "Blow-up sets and Fujita type curves for a degenerate parabolic system with nonlinear boundary conditions," Indiana University Mathematics Journal, vol. 50, no. 1, pp. 629-654, 2001.

[8] Z. Wang, J. Yin, and C. Wang, "Large-time behaviour of solutions to non-Newtonian filtration equations with nonlinear boundary sources," Proceedings of the Royal Society of Edinburgh A. Mathematics, vol. 140, no. 4, pp. 833-855, 2010.

[9] Z. Wang, J. Yin, and C. Wang, "Critical exponents of the non-Newtonian polytropic filtration equation with nonlinear boundary condition," Applied Mathematics Letters, vol. 20, no. 2, pp. 142-147, 2007.

[10] C. Jin and J. Yin, "Critical exponents and non-extinction for a fast diffusive polytropic filtration equation with nonlinear boundary sources," Nonlinear Analysis, vol. 67, no. 7, pp. 22172223, 2007.

[11] Z. Li and C. Mu, "Critical curves for fast diffusive nonNewtonian equations coupled via nonlinear boundary flux," Journal of Mathematical Analysis and Applications, vol. 340, no. 2, pp. 876-883, 2008.

[12] P. Y. H. Pang, Z. Wang, and J. Yin, "Critical exponents for nonlinear diffusion equations with nonlinear boundary sources," Journal of Mathematical Analysis and Applications, vol. 343, no. 2, pp. 654-662, 2008. 


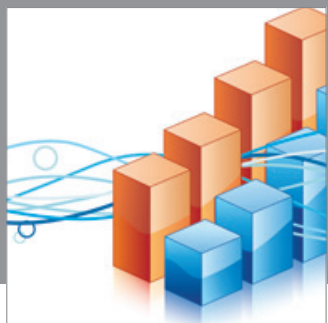

Advances in

Operations Research

mansans

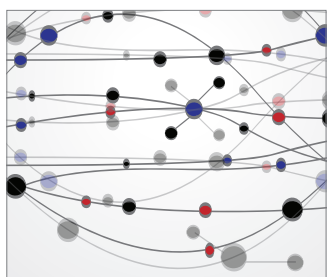

The Scientific World Journal
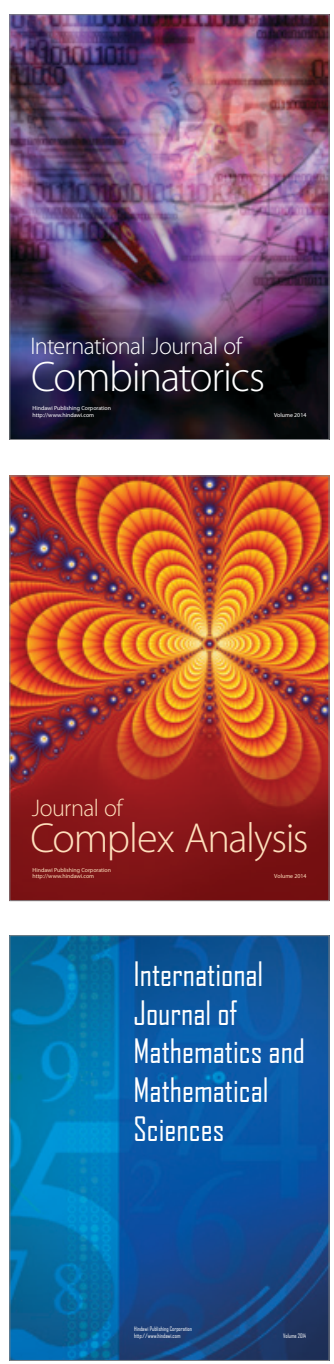
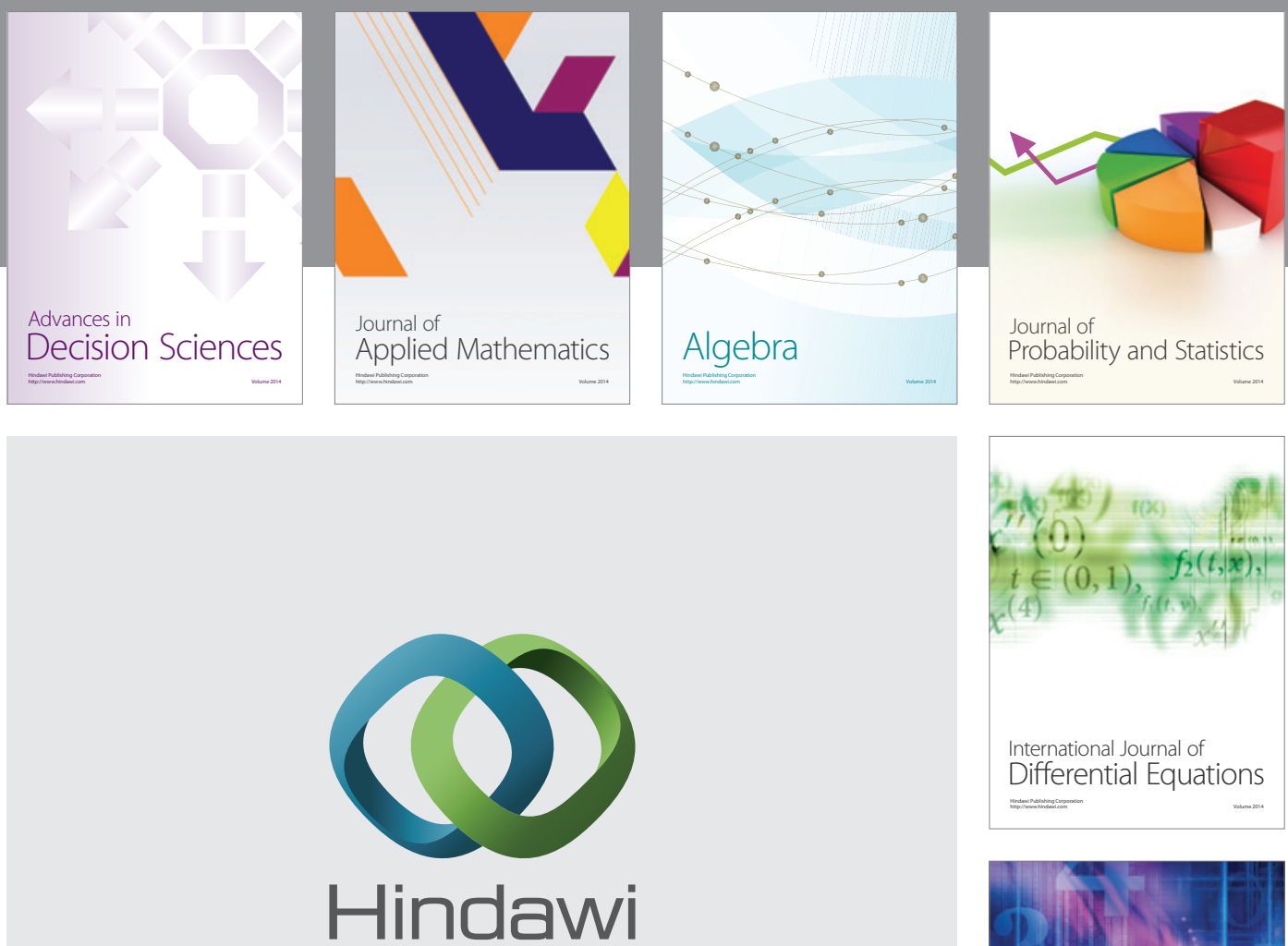

Submit your manuscripts at http://www.hindawi.com
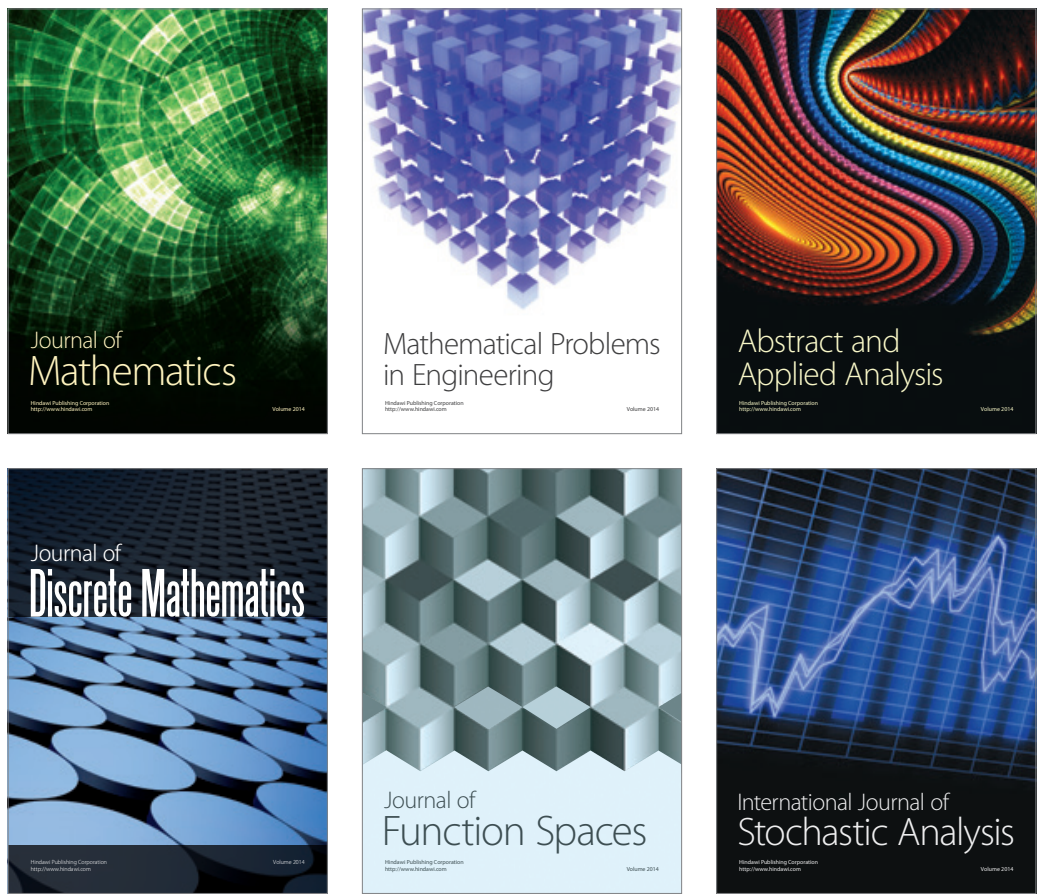

Journal of

Function Spaces

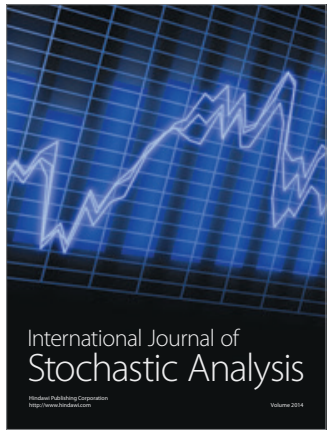

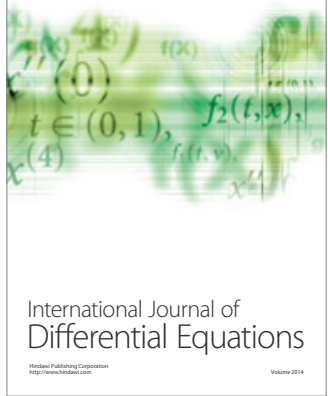
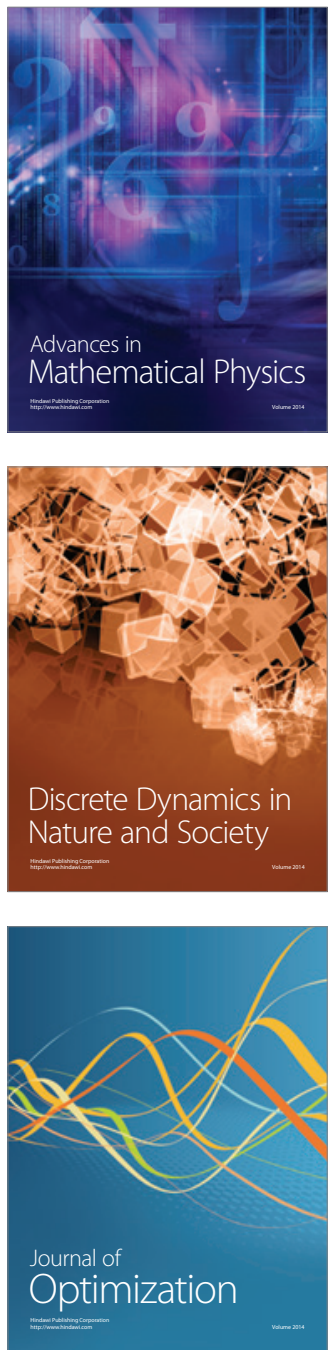